\title{
Operation of a free electron laser from the extreme ultraviolet to the water window
}

W. Ackermann, ${ }^{27}$ G. Asova,${ }^{6}$ V. Ayvazyan, ${ }^{5}$ A. Azima,${ }^{5}$ N. Baboi,${ }^{5}$ J. Bähr, ${ }^{6}$ V. Balandin, ${ }^{5}$ B. Beutner ${ }^{9}$ A. Brandt, ${ }^{5}$ A. Bolzmann, ${ }^{2}$ R. Brinkmann, ${ }^{5}$ O.I. Brovko, ${ }^{17}$ M. Castellano, ${ }^{10}$ P. Castro, ${ }^{5}$ L. Catani, ${ }^{12}$ E. Chiadroni, ${ }^{12}$ S. Choroba,${ }^{5}$ A. Cianchi, ${ }^{12}$ J.T. Costello,${ }^{22}$ D. Cubaynes,${ }^{20}$ J. Dardis, ${ }^{22}$ W. Decking,${ }^{5}$ H. Delsim-Hashemi, ${ }^{9}$ A. Delserieys, ${ }^{15}$ G. Di Pirro, ${ }^{10}$ M. Dohlus,${ }^{5}$ S. Düsterer, ${ }^{5}$ A. Eckhardt,${ }^{9}$ H.T. Edwards,${ }^{7}$ B. Faatz, ${ }^{5}$ J. Feldhaus, ${ }^{5}$ K. Flöttmann, ${ }^{5}$ J. Frisch, ${ }^{25}$ L. Fröhlich, ${ }^{9}$ T. Garvey, ${ }^{19}$ U. Gensch, ${ }^{6}$ Ch. Gerth,${ }^{5}$ M. Görler, ${ }^{5}$ N. Golubeva, ${ }^{5}$ H.-J. Grabosch, ${ }^{6}$ M. Grecki, ${ }^{26}$ O. Grimm, ${ }^{5}$ K. Hacker, ${ }^{5}{ }^{9}$ U. Hahn, ${ }^{5}$ J.H. Han, ${ }^{5}$ K. Honkavaara, ${ }^{9}$ T. Hott, ${ }^{5}$ M. Hüning, ${ }^{5}$

Y. Ivanisenko, ${ }^{18}$ E. Jaeschke, ${ }^{3}$ W. Jalmuzna, ${ }^{13}$ T. Jezynski, ${ }^{26}$ R. Kammering, ${ }^{5}$ V. Katalev, ${ }^{5}$ K. Kavanagh, ${ }^{22}$

E.T. Kennedy, ${ }^{22}$ S. Khodyachykh, ${ }^{6}$ K. Klose, ${ }^{6}$ V. Kocharyan, ${ }^{5}$ M. Körfer, ${ }^{5}$ M. Kollewe, ${ }^{5}$ W. Koprek, ${ }^{13}$ S.

Korepanov, ${ }^{6}$ D. Kostin, ${ }^{5}$ M. Krassilnikov, ${ }^{6}$ G. Kube, ${ }^{5}$ M. Kuhlmann, ${ }^{5}$ C.L.S. Lewis, ${ }^{15}$ L. Lilje, ${ }^{5}$ T. Limberg, ${ }^{5}$ D. Lipka, ${ }^{5}$ F. Löhl, ${ }^{9}$ H. Luna, ${ }^{22}$ M. Luong, ${ }^{1}$ M. Martins,,${ }^{9}$ M. Meyer, ${ }^{20}$ P. Michelato, ${ }^{11}$ V. Miltchev, ${ }^{9}$ W.D. Möller, ${ }^{5}$ L. Monaco, ${ }^{11}$ W.F.O. Müller, ${ }^{27}$ O. Napieralski, ${ }^{26}$ O. Napoly, ${ }^{1}$ P. Nicolosi, ${ }^{4}$ D. Nölle, ${ }^{5}$ T. Nuñez, ${ }^{5}$ A. Oppelt,${ }^{6}$ C. Pagani, ${ }^{11}$ R. Paparella,${ }^{1}$ N. Pchalek, ${ }^{5}, 9$ J. Pedregosa-Gutierrez,${ }^{22}$ B. Petersen, ${ }^{5}$ B. Petrosyan, ${ }^{6}$ G.

Petrosyan, ${ }^{5}$ L. Petrosyan, ${ }^{5}$ J. Pflüger,${ }^{5}$ E. Plönjes, ${ }^{5}$ L. Poletto, ${ }^{4}$ K. Pozniak, ${ }^{13}$ E. Prat,,${ }^{5}$ D. Proch, ${ }^{5}$ P. Pucyk, ${ }^{13}$ P. Radcliffe, ${ }^{5}$ H. Redlin, ${ }^{5}$ K. Rehlich, ${ }^{5}$ M. Richter, ${ }^{24}$ M. Roehrs, ${ }^{5,}{ }^{9}$ J. Roensch, ${ }^{9}$ R. Romaniuk, ${ }^{13}$ M. Ross ${ }^{25}$ J. Rossbach, ${ }^{9}$ V. Rybnikov, ${ }^{5}$ M. Sachwitz, ${ }^{6}$ E.L.Saldin, ${ }^{5}$ W. Sandner, ${ }^{21}$ H. Schlarb, ${ }^{5}$ B. Schmidt, ${ }^{5}$ M. Schmitz, ${ }^{5}$ P. Schmüser, ${ }^{9}$ J.R. Schneider, ${ }^{5}$ E.A. Schneidmiller, ${ }^{5}$ S. Schnepp, ${ }^{27}$ S. Schreiber, ${ }^{5}$ M. Seidel, ${ }^{5,23}$ D. Sertore, ${ }^{11}$ A.V. Shabunov, ${ }^{17}$ C. Simon, ${ }^{1}$ S. Simrock, ${ }^{5}$ E. Sombrowski, ${ }^{5}$ A.A. Sorokin, ${ }^{16,}{ }^{24}$ P. Spanknebel, ${ }^{8}$ R. Spesyvtsev, ${ }^{18}$ L. Staykov, ${ }^{6}$ B. Steffen, ${ }^{5}$ F. Stephan, ${ }^{6}$ F. Stulle, ${ }^{5}$ H. Thom, ${ }^{5}$ K. Tiedtke, ${ }^{5}$ M. Tischer,${ }^{5}$ S. Toleikis, ${ }^{5}$

R. Treusch, ${ }^{5}$ D. Trines,${ }^{5}$ I. Tsakov, ${ }^{14}$ E. Vogel,${ }^{5}$ T. Weiland, ${ }^{27}$ H. Weise, ${ }^{5}$ M. Wellhöfer, ${ }^{9}$ M. Wendt,${ }^{5,7} \mathrm{I}$.

Will, ${ }^{21}$ A. Winter ${ }^{5}$ K. Wittenburg, ${ }^{5}$ W. Wurth, ${ }^{9}$ P. Yeates, ${ }^{22}$ M.V. Yurkov, ${ }^{5}, *$ I. Zagorodnov, ${ }^{5}$ and K. Zapfe ${ }^{5}$

${ }^{1}$ CEA Saclay, 91191 Gif-sur-Yvette, France

${ }^{2}$ Bayerische Julius-Maximilians Universität, Inst. f. Theor. Physik u. Astrophysik, Am Hubland, 97074 Wurzburg, Germany ${ }^{3}$ BESSY GmbH, Albert-Einstein-Str.15, 12489 Berlin, Germany

${ }^{4}$ CNR-INFM, Dep. of Information Engineering,

University of Padova, via Gradenigo 6/B, 35131, Padova, Italy

${ }^{5}$ Deutsches Elektronen-Synchrotron DESY, Notkestrasse 85, 22603 Hamburg, Germany

${ }^{6}$ Deutsches Elektronen-Synchrotron DESY, Platanenallee 6, 15738 Zeuthen, Germany

${ }^{7}$ Fermi National Accelerator Laboratory, MS 306, P.O.Box 500, Batavia, IL 60510 USA

${ }^{8}$ Humboldt Universität, Unter den Linden 6, 10099 Berlin, Germany

${ }^{9}$ Universität Hamburg, Inst. f. Experimentalphysik,

Luruper Chaussee 149, 22761 Hamburg, Germany

${ }^{10}$ INFN-LNF, via E. Fermi 40, 00044 Frascati, Italy

${ }^{11}$ INFN Milano-LASA, via Fratelli Cervi 201,l 20090 Segrate (MI), Italy

${ }^{12}$ INFN-Roma Tor Vergata, Via della Ricerca Scientifica, 1, 00133 Rome, Italy

${ }^{13}$ Institute of Electronic Systems, Warsaw University of Technology, Nowowiejska 15/19, Warsaw, Poland

${ }_{14}^{14}$ Institute for Nuclear Researches and Nuclear Energy,

Tzarigradsko Shaussee Boulevard 72, 1784 Sofia, Bulgaria

${ }^{15}$ International Research Centre for Experimental Physics, Queen's University, Belfast, BT7 1NN, UK

${ }^{16}$ Ioffe Physico-Technical Institute RAS, Polytekhnicheskaya 26, 194021 St.Petersburg, Russia

${ }^{17}$ Joint Institute for Nuclear Research, 141980 Dubna, Moscow Region, Russia

${ }^{18}$ Kharkiv National University, 4 Svobody sq, 61077, Kharkiv, Ukraine

${ }^{19}$ Laboratoire de L'Accelerateur Lineaire, IN2P3-CNRS,

Universite de Paris-Sud, B.P. 34, 91898 Orsay, France

${ }^{20}$ LIXAM/CNRS, Centre Universitaire Paris-Sud, Batiment 350, F-91405 Orsay Cedex, France

${ }^{21}$ Max-Born-Institute, Max-Born-Str. 2a, 12489 Berlin, Germany

${ }^{22}$ National Center for Plasma Science and Technology and School for Physical Sciences, Dublin City University, Dublin, Ireland

${ }^{23}$ Paul Scherrer Institute, 5232 Villigen PSI, Switzerland

${ }^{24}$ Physikalisch-Technische Bundesanstalt, PTB, Abbestrasse 2-12, D-10587 Berlin, Germany

${ }^{25}$ Stanford Linear Accelerator Center, 2575 Sand Hill Road, Menlo Park, CA 94025, USA

${ }^{26}$ Technical University of Lodz, Al. Politechniki 11, Lodz, Poland

${ }^{27}$ Technische Universität Darmstadt, FB 18, Institut TEMF,

Schlossgartenstr. 8, 64289 Darmstadt, Germany

(Dated: as accepted for publication after peer review)

We report results on the performance of a free electron laser operating at a wavelength of $13.7 \mathrm{~nm}$ where unprecedented peak and average powers for a coherent EUV radiation source have been measured. In the saturation regime the peak energy approached $170 \mu \mathrm{J}$ for individual pulses while 
the average energy per pulse reached $70 \mu \mathrm{J}$. The pulse duration was in the region of 10 femtoseconds and peak powers of $10 \mathrm{GW}$ were achieved. At a pulse repetition frequency of 700 pulses per second, the average EUV power reached $20 \mathrm{~mW}$. The output beam also contained a significant contribution from odd harmonics of approximately $0.6 \%$ and $0.03 \%$ for the $3 \mathrm{rd}(4.6 \mathrm{~nm})$ and the 5 th $(2.75 \mathrm{~nm})$ harmonics, respectively. At 2.75 nanometers the 5 th harmonic of the radiation reaches deep into the water window - a wavelength range that is crucially important for the investigation of biological samples.

The generation of laser-like radiation in the extremeultraviolet (EUV) spectral range has been a grand challenge for scientists and engineers dating back to the very earliest days of the laser in the 1960s. Impressive progress was made in the 1980s when lasing in the EUV via population inversion between levels in highly charged ions was observed in laser plasma experiments [1, 2]. Work on these sources has undergone continuous development since then, even including a seeding option [3]. High harmonics of optical lasers were also taking hold around that time as sources of coherent EUV radiation $[4,5]$. Indeed, harmonics up to very high order have been generated from solids in recent times [6]. An alternative approach and one that is now beginning to pay rich dividends is the production of laser quality radiation using free electrons accelerated to relativistic speeds in linear accelerators.

Recent advances in accelerator and precision magnetic undulator technologies, now make possible the construction of single pass Free Electron Lasers (FELs) based on self-amplified spontaneous emission (SASE) where the light amplification process starts from shot noise in the electron beam [7-9]. These new sources provide uniquely intense, polarized, short-pulse radiation tunable throughout the VUV and X-ray wavelength range with a brilliance which exceeds both modern synchrotron radiation and laser plasma sources by many orders of magnitude in peak and average brilliance (see Fig. 1).

The first demonstration of the SASE FEL mechanism took place in 1997 and concerned lasing in the infrared wavelength range [10]. In September 2000, a group at Argonne National Laboratory (ANL) became the first to demonstrate saturation in a visible $(390 \mathrm{~nm})$ SASE FEL [11]. Soon after, in September 2001, a group at DESY (Hamburg, Germany) demonstrated lasing to saturation deep into the vacuum-UV at $98 \mathrm{~nm}[12,13]$. Since August 2005 the latest generation SASE FEL FLASH (Free Electron Laser in Hamburg) has been operating as a user facility [14] providing radiation at a fundamental wavelength which can be tuned from $47 \mathrm{~nm}$ down to $13 \mathrm{~nm}$. The facility is hosting many international groups exploiting the unique aspects of this source in projects ranging from atomic physics through materials science to biology [15-19]. After the energy upgrade of the FLASH linac to $1 \mathrm{GeV}$ in 2007 , it will be possible to generate wavelengths down to $6 \mathrm{~nm}$ in the fundamental harmonic.

Recently the German government approved $60 \%$ of the funding for a hard X-ray SASE FEL user facility - the European X-Ray Free Electron Laser (XFEL) [20, 21],

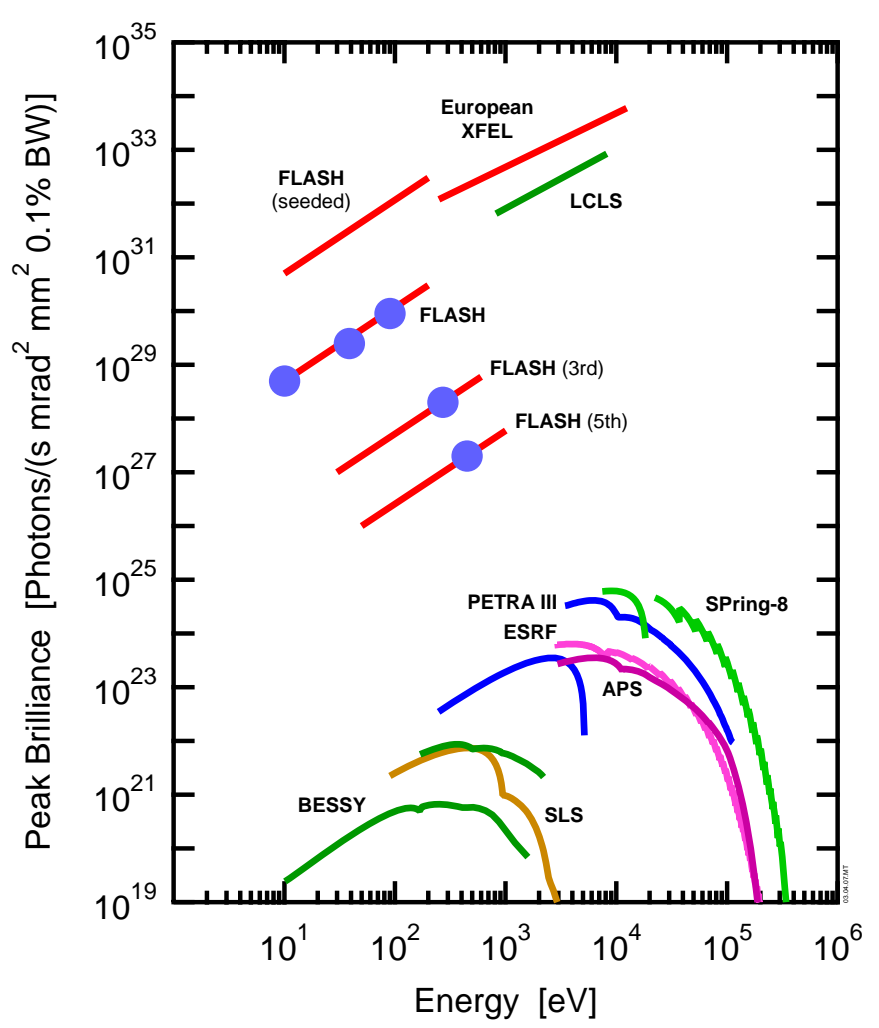

FIG. 1: Peak brilliance of X-ray FELs versus 3rd generation SR light sources. Blue spots show experimental performance of the FLASH free electron laser at DESY at the fundamental, $3 \mathrm{rd}$, and 5th harmonics.

the US Department of Energy (DOE) has approved the start of construction of the Linac Coherent Light Source (LCLS) at SLAC [22], and the project of the SPring8 Compact SASE Source (SCSS) is under development in Japan [23]. LCLS and SCSS will employ normalconducting, room temperature linacs while the European XFEL will utilize superconducting accelerator technology. LCLS has entered the construction phase with an expected completion date in 2008 . It will be ready to host users in 2009. The expected commissioning phase for the SCSS is set for 2010, and the European XFEL will start operation in 2013. FLASH is the precursor for the European XFEL and so it is a key platform technology on the blueprint for large scale X-ray FELs.

Brilliance, coherence, and timing down to the femtosecond regime are the three properties which provide the potential for new science to be explored with EUV 
and X-ray FELs. Since SASE FELs can produce pulses with unprecedented peak intensity, they open up the vista of EUV and X-ray non-linear optics and spectroscopy. The primary interaction is with inner shell electrons and so EUV laser matter interactions will be a rich vein of new science and applications in the coming decade. The first experimental results demonstrating the ability of future XFELs to generate pulses of a few tens of femtoseconds have been achieved recently at FLASH [12-14].

The timing of this report is appropriate for several reasons. A crucial milestone on the X-ray FEL roadmap was reached recently when FLASH produced saturated laserlike EUV radiation pulses at $13.7 \mathrm{~nm}$ for the first time. This $13 \mathrm{~nm}$ region is important because of its relevance to EUV lithography. At saturation, FLASH delivers ultrashort pulses with durations as low as $10 \mathrm{fs}$ and with peak and average powers of up to $10 \mathrm{GW}$ and $20 \mathrm{~mW}$ respectively - record values for EUV lasers. FLASH also produces bright emission at the third harmonic $(4.6 \mathrm{~nm})$ and the fifth harmonic $(2.75 \mathrm{~nm})$ of the fundamental mode. The latter wavelength is shorter than any produced to date by plasma based X-ray lasers and it lies well within in the so-called water window where biological systems can be imaged and analyzed in vitro (and potentially in vivo). In addition, the pulse durations of the harmonics decrease with harmonic number and so their durations lie in the single digit femtosecond range opening up the possibility to study deep inner shell atomic and molecular dynamics on a subfemtosecond timescale.

\section{RESULTS}

\section{Production of electron bunches}

FLASH is a SASE FEL that produces EUV radiation during a single-pass of an electron beam through a long periodic magnetic undulator [7-9]. The driving mechanism of a free electron laser is the radiative instability of the electron beam due to the collective interaction of electrons with the electromagnetic field in the undulator [24]. The amplification process in SASE FELs starts from the shot noise in the electron beam. When the electron beam enters the undulator, the induced beam modulation at wavelengths close to the resonance wavelength

$$
\lambda=\lambda_{\mathrm{w}}\left(1+K^{2}\right) /\left(2 \gamma^{2}\right)
$$

initiates the process of radiation emission (here $\lambda_{\mathrm{w}}$ is the undulator period, $K=e B_{\mathrm{w}} \lambda_{\mathrm{w}} / 2 \pi m_{\mathrm{e}} c$ is the undulator parameter, $B_{\mathrm{w}}$ is the rms value of the undulator field, $\gamma$ is the relativistic factor, $c$ is the velocity of light, and $m_{\mathrm{e}}$ and $e$ are the mass and charge of the electron, respectively). The interaction between the electrons oscillating in the undulator and the radiation that they produce, leads to a periodic longitudinal density modulation (microbunching) with a period equal to the resonance wavelength. The radiation emitted by the microbunches is in phase and adds coherently leading to an increase in the photon intensity which further enhances the microbunching. The amplification process develops exponentially with the undulator length and an intensity gain in excess of $10^{7}$ is obtained in the saturation regime. At this level, the shot noise of the electron beam is amplified up to the point at which complete micro-bunching is achieved and almost all electrons radiate in phase producing powerful, coherent radiation.

A qualitative estimation of the FEL operating parameter space can be obtained in terms of the FEL parameter $\rho[25]$ :

$$
\rho=\left[\frac{I}{I_{\mathrm{A}}} \frac{A_{J J}^{2} K^{2} \lambda_{\mathrm{w}}^{2}}{32 \pi^{2} \gamma^{2} \sigma_{\perp}^{2}}\right]^{1 / 3} .
$$

Here $I$ is the beam current, $I_{\mathrm{A}}=17 \mathrm{kA}$ is the Alfven current, $\sigma_{\perp}$ is the r.m.s. transverse size of the electron bunch, the coupling factor is $A_{\mathrm{JJ}}=1$ for helical undulator and $A_{\mathrm{JJ}}=\left[J_{0}(Q)-J_{1}(Q)\right]$ for planar undulator, $Q=K^{2} /\left[2\left(1+K^{2}\right)\right]$, and $J_{0}$ and $J_{1}$ are the Bessel functions of the first kind.

Estimates for the main FEL parameters are as follows: the field gain length is about $L_{\mathrm{g}} \simeq \lambda_{\mathrm{w}} /(4 \pi \rho)$, the FEL efficiency in the saturation regime is approximately equal to $\rho$, the spectral bandwidth is approximately $2 \rho$, and the coherence time is $\tau_{\mathrm{c}} \simeq L_{\mathrm{g}} \lambda /\left(\lambda_{\mathrm{w}} c\right)$.

The FLASH facility has already been described in detail elsewhere [14]. A comprehensive description of specific systems, with relevant references, is presented in the Supplementary Information, sections 1-3. Figure 2 (top) shows the schematic layout of the FLASH facility. The electron beam is produced in a radio frequency gun and brought up to an energy of $700 \mathrm{MeV}$ by five accelerating modules ACC1 to ACC5 [14]. At energies of 130 and 380 $\mathrm{MeV}$ the electron bunches are compressed in the bunch compressors BC1 and BC2. The undulator is a fixed 12 mm gap permanent magnet device with a period length of $2.73 \mathrm{~cm}$ and a peak magnetic field of $0.47 \mathrm{~T}$. The undulator system is subdivided into six segments, each 4.5 $\mathrm{m}$ long.

The electron beam formation system is based on the use of nonlinear longitudinal compression. When the bunch is accelerated off-crest in the accelerating module, the longitudinal phase space acquires a radio frequency induced curvature. Downstream of each bunch compressor, this distortion results in a non-Gaussian distribution within the bunch and in a local charge concentration. It is the leading edge of the bunch, with its high peak current, which is capable of driving the high intensity lasing process (see Fig. 2). With proper optimization of the 

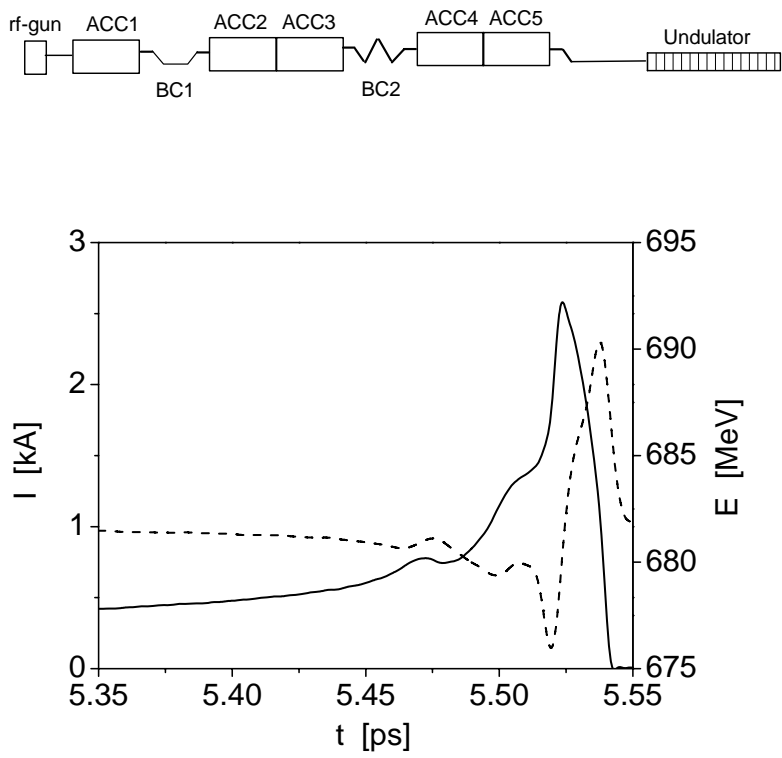

FIG. 2: Production of electron bunches at FLASH. Top: Schematic layout of the FLASH facility. Abbreviations ACC and $\mathrm{BC}$ stand for accelerating module and bunch compressor, respectively. The electron beam originates from the laserdriven radio frequency gun, is accelerated and compressed to a high value of peak current in the magnetic compressors. Bottom: current $I$ (solid line) and mean energy $E$ (dashed line) along the electron bunch at the undulator entrance (simulation). The bunch head is located at the right hand side of the figure.

bunch compression system it is possible to obtain a low transverse emittance for the high-current spike which is absolutely crucial for the production of high quality FEL beams. In this regard, it should be noted that collective effects play a significant role in the bunch compression process for short pulses. In the high-current part of the bunch, with rms length $\sigma_{z}$ and peak current $I$, coherent synchrotron radiation (CSR) and longitudinal space charge (LSC) effects scale as $I / \sigma_{z}^{1 / 3}$ and $I / \sigma_{z}$, respectively. For instance, the LSC-induced energy chirp along the high-current spike of the bunch grows when the bunch travels from the bunch compressor to the undulator entrance [26]:

$$
\frac{d(\Delta \gamma)}{d z} \simeq 2.4 \frac{I}{I_{\mathrm{A}}} \frac{\ln \left(\gamma \sigma_{z} / \sigma_{\perp}\right)}{\sigma_{z} \gamma^{2}} .
$$

One can see the power of this effect in Fig. 2 where an increase in the peak current and a narrowing of the width of the spike leads to an increase in the induced energy chirp due to space charge effects. For FLASH, operating at a wavelength of $13 \mathrm{~nm}$, the value of the FEL parameter is $\rho=(2.5-3) \times 10^{-3}$. The lasing portion of the electron bunch possesses a strong energy chirp (see Fig. 2 lower panel) which results in an additional broadening of the FEL spectral distribution. Another consequence of the energy chirp is suppression of the FEL gain. The effect of the energy chirp on the gain is given by the parameter $\left(\rho \gamma / \tau_{\mathrm{c}}\right)^{-1} \mathrm{~d} \gamma / \mathrm{d} t$ and it starts to play a significant role when the relative energy change acquired within one coherence length $c \tau_{\mathrm{c}}$, becomes comparable with the FEL parameter $\rho[27,28]$. This effect is strong in the case under study here and results in a significant correction to the FEL gain. Finally, the effect of the energy chirp leads to further shortening of the lasing part of the electron bunch (and hence the FEL pulse length) in the regime of exponential growth.

\section{Saturated output and gain length}

To date FLASH has demonstrated its ability to generate powerful, coherent, continuously tuneable radiation in the wavelength range from 47 to $13 \mathrm{~nm}$ at the fundamental harmonic, and down to $2.75 \mathrm{~nm}$ at the 5 th harmonic. In this paper we present a thorough measurement and characterization of the properties of the FEL radiation at a wavelength of $13.7 \mathrm{~nm}$. The average energy in the radiation pulse demonstrated at this wavelength was $70 \mu \mathrm{J}$, although the characterization reported here was performed at an average energy of $40 \mu \mathrm{J}$. Figure 3 shows the spatial profile of the FEL radiation detected on a Ce:YAG screen located $23.5 \mathrm{~m}$ downstream of the undulator exit. The FWHM spot size is $2.1 \mathrm{~mm}$ which corresponds to an angular divergence of $90 \pm 10 \mu \mathrm{rad}$ (FWHM). The radiation mode in the far field is nearly axisymmetric. With the additional knowledge of the EUV spot size at the undulator exit (ca. $160 \mu \mathrm{m}$ FWHM), we find that the product of the spot size of the radiation at the undulator exit and the angular divergence results in a value of about the radiation wavelength. This means that the phase volume of the radiation is close to the diffraction limit, and that the radiation has a high degree of transverse coherence.

We present in Fig. 4a the average EUV pulse energy versus undulator length where the exponential growth from spontaneous radiation to saturated laser output can be seen. Each point in this figure was obtained by averaging over 300 pulses (see Supplementary Information, section 5 for more details). The radiation energy was measured with a MCP based detector [29] which was operated with a $5 \mathrm{~mm}$ diameter aperture and was located $18.5 \mathrm{~m}$ from the undulator. The interaction length in the undulator (and hence amplification) was changed by means of a transverse kick of the electron beam trajectory between the undulator modules which is strong enough to stop the FEL amplification process downstream of the orbit kick. With the FEL interaction suppressed along the whole length of the undulator chain, the residual spontaneous emission from the full undulator length and also the full electron bunch was measured. The FEL interaction was switched on gradually along the undulator and the en- 

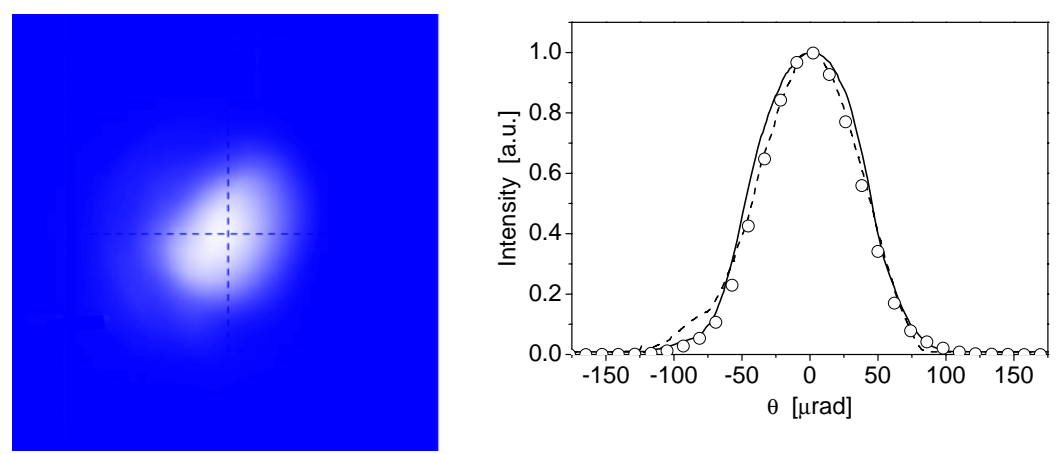

FIG. 3: Spatial profile of the FEL radiation. Left: photon beam image on a Ce:YAG crystal averaged over many shots. The Ce:YAG screen is located $23.5 \mathrm{~m}$ downstream of the undulator exit. The FWHM spot size is $2.1 \mathrm{~mm}$. Right: vertical (solid line) and horizontal (dashed line) slices of the image. Circles represent simulation results with the code FAST [38]. The average energy in the radiation pulse was $40 \mu \mathrm{J}$. The radiation wavelength was $13.7 \mathrm{~nm}$.
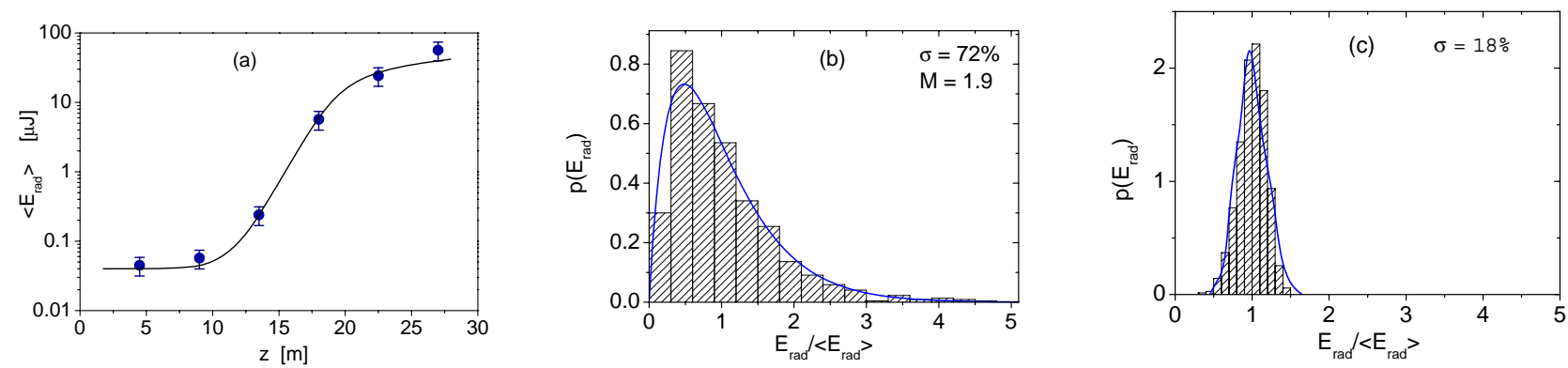

FIG. 4: Energy in the radiation pulse and its fluctuations. Left: average energy in the radiation pulse versus undulator length showing exponential growth and saturation. Error bars correspond to the uncertainty in the calibration of the MCP-detector of $\pm 15 \%$. Center and rightmost panels show the probability distributions for the energy in the radiation pulses for the end of the regime of exponential growth and for the saturation regime, respectively. Radiation wavelength is $13.7 \mathrm{~nm}$. Solid line on the center plot represents a gamma distribution (4) with the parameter $M=1.9$. The solid line on the right plot represents simulations with the code FAST [38].

ergy in the radiation pulse grew steadily. Independent measurements of the radiation energy at saturation were made with the aid of the gas monitor detector [30]. Analysis of the exponential part of the gain curve presented in Fig. 4a yields a field gain length of $L_{\mathrm{g}}=2.5 \pm 0.3 \mathrm{~m}$. This measurement gives us an estimate for the coherence time $\tau_{\mathrm{c}} \simeq 4.2 \pm 0.5 \mathrm{fs}$.

\section{EUV pulse duration}

The energy of each EUV pulse fluctuates from shot to shot since the lasing process starts up from shot noise. When the FEL amplification process takes place, fluctuations in the EUV field grow with undulator length and reach a maximum value at the end of the regime of exponential growth just before saturation. When the amplification process finally reaches saturation, the fluctuations drop sharply. The measured probability distributions of the radiation energy for these two regimes are shown in
Fig. $4 \mathrm{~b}$ and $4 \mathrm{c}$ (see Supplementary Information, section 5 for more details). Data for the exponential and the saturation regime were taken at the average energy in the radiation pulse of 1 and $40 \mu \mathrm{J}$, respectively. For a SASE FEL operating in the regime of exponential growth it is well known that the radiation exhibits the properties of completely chaotic polarized light [31]. One consequence is that the probability distribution of the energy in the radiation pulse fits to a gamma-distribution:

$$
p(E)=\frac{M^{M}}{\Gamma(M)}\left(\frac{E}{\langle E\rangle}\right)^{M-1} \frac{1}{\langle E\rangle} \exp \left(-M \frac{E}{\langle E\rangle}\right),
$$

where $\Gamma(M)$ is the gamma function, $M=1 / \sigma_{E}^{2}$, and $\sigma_{E}^{2}=\left\langle(E-\langle E\rangle)^{2}\right\rangle /\langle E\rangle^{2}$. The parameter $M$ can be interpreted as the average number of "degrees of freedom" or "modes" in the radiation pulse. Thus, from the experimental result for $M=1.9$, and an estimate for the coherence time $\tau_{\mathrm{c}} \simeq 4 \mathrm{fs}$, we come to an estimate for the radiation pulse length in the end of the regime of 

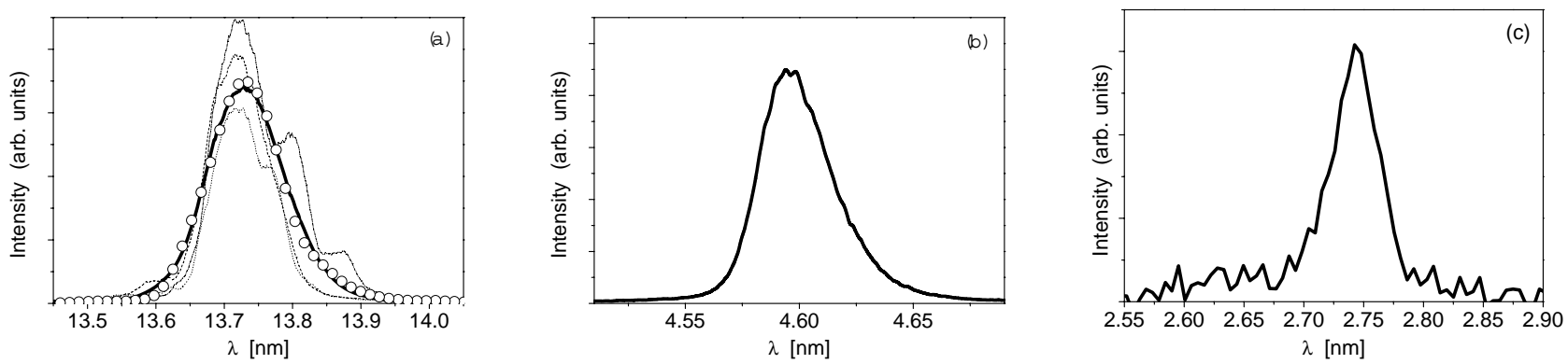

FIG. 5: EUV spectra of the fundamental (left), 3rd harmonic (center) and 5th harmonic (right) contributions to the FEL output. Bold lines show averaged spectra (mean value of 300 shots) while thin lines show single-shot spectra. Circles on the left plot represent averaged spectra simulated with the code FAST [38]. The average energy in the FEL pulse is $40 \mu \mathrm{J}$.

exponential growth of about $8 \pm 1$ fs.

\section{Spectral distribution of the FLASH output}

Results of spectral measurements are presented in Fig. 5. During these measurements FLASH operated in the saturation regime with an average energy in the radiation equal to $40 \mu \mathrm{J}$. Single-shot spectra were obtained with the plane grating monochromator, operating in spectographic mode, with a resolving power $\lambda / \Delta \lambda$ of 1500 [32]. The bold curve in Fig. 5a represents the average value of 300 pulses - single shots are also shown as light curves. We observe that the single-shot spectra are dominated by a single feature, albeit accompanied in some cases by some small modulations. Since the spectrum is simply a Fourier transform of the temporal structure, we conclude that the temporal profile of the radiation pulses also consists mostly of a single spike. This qualitative observation is in a good agreement with the measured number of modes $M=1.9$ (see Fig. 4b) which tells us that the radiation has nearly complete longitudinal coherence. This conclusion is also supported by significant suppression of the measured fluctuations of the radiation energy when spectrally filtered by a narrow band monochromator (see Supplementary Information, section 6 for more details).

Another observation is the rather large width of the averaged spectrum, about $1 \%$ of the EUV wavelength. Our analysis shows that half of this value comes from the amplification bandwidth and the additional broadening is due to a strong energy chirp along the lasing spike (see Fig. 2).

\section{Harmonics of the $13.7 \mathrm{~nm}$ fundamental mode}

Radiation from a SASE FEL operating at saturation also contains relatively strong contributions from higher frequency harmonics [33-35]. Figure 5b shows average spectrum of the 3rd harmonic. Comparison of the spec- trum of the fundamental and the 3rd harmonic shows that the relative bandwidth, $\Delta \lambda / \lambda_{h}$, remains nearly constant, a result which is in good agreement with theoretical predictions for the properties of higher harmonics generated in the SASE FEL operating in the saturation regime [36]. Note that in the case of ordinary undulator radiation, the relative spectral width, $\Delta \lambda / \lambda_{h}$ scales inversely with the harmonic number $h$. The spectrum of the 5th harmonic is shown on Fig. 5c. The spectrum was obtained by averaging more than 1000 pulses as the grating and mirror reflectivities were very small at this wavelength.

For an average EUV pulse energy of $40 \mu \mathrm{J}$ at the fundamental wavelength, we measured $0.25 \pm 0.1 \mu \mathrm{J}$ for the $3 \mathrm{rd}(4.6 \mathrm{~nm})$ and $10 \pm 4 \mathrm{~nJ}$ for the 5 th $(2.75 \mathrm{~nm})$ harmonic. These values correspond to a relative contribution to the total EUV output energy of $0.6 \pm 0.2 \%$ and $0.03 \pm 0.01 \%$ for the 3rd and the 5th harmonics which is in agreement with prediction [36]. The physical mechanism underlying the generation of higher harmonics is a nonlinear transformation which results in a shortening of the FEL pulse width. With a 10 fs pulse duration for the fundamental harmonic, we estimate peak powers of $40 \mathrm{MW}$ and $2 \mathrm{MW}$ for the 3rd and the 5th harmonics respectively. The corresponding average powers were approximately 120 microwatts and 6 microwatts. Hence the available intensities are sufficient for performing experiments in the so-called "water window" - a wavelength range that is crucially important for the investigation of biological samples.

Theoretical predictions tell us that the contribution of the 2nd harmonic to the total radiation power depends strongly on the ratio of the FEL gain length to the Rayleigh length of the radiation [37]. For a ratio of unity, the 2nd harmonic intensity comes out to be a fraction of a per cent and decreases rapidly as this ratio increases. The estimated ratio for the parameter range of FLASH operating at the wavelength of $13.7 \mathrm{~nm}$ is about ten. Measurements of the even harmonics have shown that they are significantly suppressed with respect to the odd harmonics. For instance, the intensity of the 2nd 


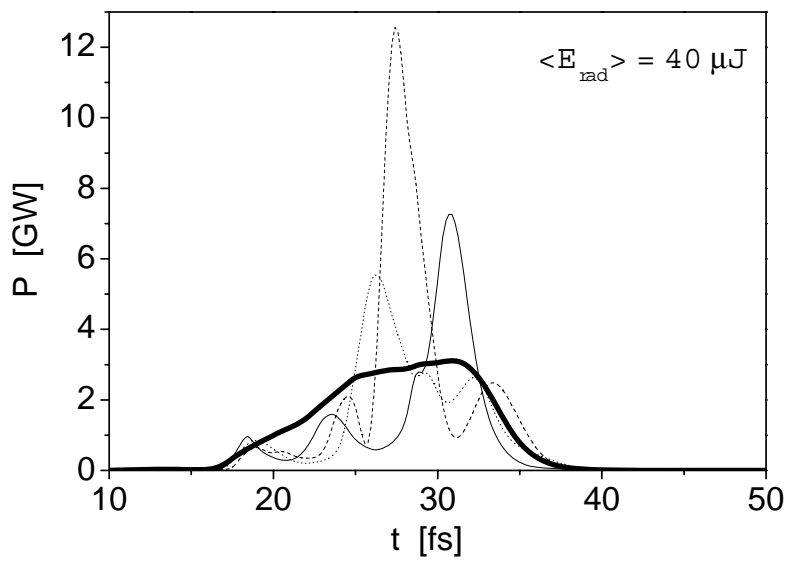

FIG. 6: The temporal structure of the radiation pulse for an average energy of $40 \mu \mathrm{J}$, predicted by the code FAST [38] using experimentally determined radiation properties. The radiation wavelength is $13.7 \mathrm{~nm}$. The bold line shows the envelope while the thin lines are single shots.

harmonic is more than an order of magnitude less than that of the 3rd harmonic.

With the measured properties of the radiation (gain curve, pulse energy, spectra, statistical properties, and spatial properties of the radiation) we can now determine the range of electron beam parameters related to the lasing fraction of the electron bunch. The electron bunch shape and energy chirp is close to that shown in Fig. 2 with a peak current of 2 to $2.5 \mathrm{kA}$. The FWHM length of the high current spike is approximately $30 \mathrm{fs}$ and the normalized emittance is 1 to $1.5 \mathrm{~mm}$-mrad. The excellent agreement between experiment and simulation with the code FAST [38] (see Figs. 3-5) allows us to specify those parameters of the radiation which cannot be measured directly. For instance, in Fig. 6 we present the temporal profile of the radiation pulse deduced from the same simulation data. We see that indeed the pulse duration is about 10 fs in a good agreement with the experimentally deduced result. The mean value of the peak power in the radiation pulse is more than $3 \mathrm{GW}$, while for some individual high energy pulses the peak power approaches 10 GW.

\section{DISCUSSION}

An important lesson from FLASH is that GW-level, laser-like EUV radiation pulses on a 10 fs scale can be produced with a reliable single-pass SASE FEL scheme. FLASH has produced unprecedented powers for EUV radiation at a fundamental wavelength of $13.7 \mathrm{~nm}$, and harmonics with wavelengths as low as $2.75 \mathrm{~nm}$ (i.e., in the range of a soft X-ray FEL). The experimental measurements show that FLASH is now operating at its ultimate performance level with a peak brilliance of $(6 \pm 3) \times 10^{29},(2 \pm 1) \times 10^{28}$, and $(2 \pm 1) \times$ $10^{27}$ phot. $/ \mathrm{sec} / \mathrm{mrad}^{2} / \mathrm{mm}^{2} /(0.1 \%$ bandwidth $)$ at 13.7 , 4.6, and $2.75 \mathrm{~nm}$, respectively (see Fig. 1). At the 5th harmonic wavelength FLASH is already approaching the wavelength range of the European XFEL and LCLS, albeit with lower brilliance. However, it is still higher than the peak brilliance of the 3rd generation SR sources by quite a few orders of magnitude.

\section{METHODS}

\section{EUV diagnostics}

The FEL beam diagnostics have already been described in detail elsewhere (see [39] and the Supplementary Information, section 4) and so only brief summary is given here. The FEL is currently operated at $5 \mathrm{~Hz}$ in either single-bunch or in multi-bunch mode. In the latter, the FEL typically produces up to 140 radiation pulses (five times per second). The FEL pulse energies were measured using microchannel plates (MCPs) [29] and gas monitor detectors [30]. The EUV spectra of the FEL fundamental mode and its harmonics were measured using three different grazing incidence spectrometer systems. The first spectrometer is described in detail in [40]. Briefly, the system contains a spherical variable line spacing grating which provides a flat field in the focal plane for easy matching to a flat detector array combined with a spherical mirror in a Kirkpatrick-Baez configuration resulting in a stigmatic spectrometer. The spectral resolution, $\lambda / \Delta \lambda$, is better than 1500 across the 3 to $40 \mathrm{~nm}$ operating wavelength range. The detection system consists of a phosphor faceplate for EUV to optical image down-conversion with a readout by a lens coupled intensified CCD camera. The second spectrometer is a plane grating monochromator equipped with a 200 lines $/ \mathrm{mm}$ grating for the photon wavelength range of 6 to $60 \mathrm{~nm}$, and a high resolution 1200 lines/mm grating for the energy range of 2 to $12 \mathrm{~nm}$ [32]. These two systems were used to measure spectra of the fundamental and the third harmonic FEL pulses and, with their gated readout, they could select a single pulse from a FEL pulse train. The third monochromator was a flat field system, equipped with a Harada grating [41] but with a highly sensitive back illuminated CCD camera (Andor Technology) containing $2048 \times 512$ pixels, each with a footprint of $13 \times 13$ microns squared. The system was operated without an entrance slit which resulted in a spectral resolution of approximately 100. A 1.5 micron thick aluminum foil placed over the entrance aperture provided a transmission window of $1 \mathrm{~nm}$ FWHM centered at about $2.8 \mathrm{~nm}$ when combined with the reflectivity of the beamline mir- 
rors. This system was used to measure the spectrum of the 5th harmonic.

\section{ACKNOWLEDGEMENT}

The authors are indebted to invaluable support by the technical staff of DESY and participating groups from the TESLA Technical Collaboration.

Supplementary information is available on the Nature Photonics website.

\section{COMPETING FINANCIAL INTERESTS}

The authors declare that they have no competing financial interests.

\section{REFERENCES}

* Corresponding author. E-mail:Mikhail.Yurkov@desy. de (M.V.Yurkov)

1. Matthews, D.L. et al. Demonstration of a soft x-ray amplifier. Phys. Rev. Lett. 54, 110-113 (1985).

2. MacGowan, B.J. et al. Demonstration of soft x-ray amplification in nickel-like ions. Phys. Rev. Lett. 59, 2157-2160 (1987).

3. Zeitoun, Ph. et al. A high-intensity highly coherent soft $\mathrm{X}$-ray femtosecond laser seeded by a high harmonic beam. Nature 431, 426-429 (2004).

4. Ferray, M. et al. Multiple-harmonic conversion of $1064 \mathrm{~nm}$ radiation in rare gases. J. Phys. B 21, L31-L33 (1988).

5. Li, X.F., LHuillier, A., Ferray, M., Lompre', L.A. \& Mainfray, G. Multiple-harmonic generation in rare gases at high laser intensity. Phys. Rev. A 39, 5751-5761 (1989).

6. Dromey, B. et al. High harmonic generation in the relativistic limit. Nature Physics, 2, 456-459 (2006).

7. Kondratenko, A.M. \& Saldin, E.L. Generation of coherent radiation by a relativistic electron beam in an undulator. Part. Accelerators 10, 207-216 (1980).

8. Derbenev, Ya.S., Kondratenko, A.M. \& Saldin, E.L. On the possibility of using a free-electron laser for polarization of electrons in storage rings. Nucl. Instrum. and Methods 193, 415-421 (1982).

9. Murphy, J.B. \& Pellegrini, C. Free electron lasers for the XUV spectral region. Nucl. Instrum. and Methods A 237, 159-167 (1985).

10. Hogan, M. et al. Measurements of gain larger than $10^{5}$ at $12 \mu \mathrm{m}$ in a self-amplified spontaneous-emission freeelectron laser. Phys. Rev. Lett. 81, 4867-4870 (1998).

11. Milton, S.V. et al. Exponential gain and saturation of a self-amplified spontaneous emission free-electron laser. Science 292, 2037-2041 (2001).

12. Ayvazyan, V, et al. Generation of GW radiation pulses from a VUV free-electron laser operating in the femtosecond regime. Phys. Rev. Lett. 88, 104802 (2002).
13. Ayvazyan, V, et al. A new powerful source for coherent VUV radiation: Demonstration of exponential growth and saturation at the TTF free-electron laser. Eur. Phys. J. D 20, 149-156 (2002).

14. Ayvazyan, V, et al. First operation of a free-electron laser generating GW power radiation at $32 \mathrm{~nm}$ wavelength. Eur. Phys. J. D 37, 297-303 (2006).

15. Wabnitz, H. et al. Multiple ionization of atom clusters by intense soft X-rays from a free-electron laser. Nature $\mathbf{4 2 0}$, 482-485 (2002).

16. Meyer, M. et al. Two-color photoionization in xuv freeelectron and visible laser fields. Phys. Rev. A 74, 011401 (2006).

17. Sorokin, A.A., Bobashev, S.V., Tiedtke. K. \& Richter, M. Multi-photon ionization of molecular nitrogen by femtosecond soft x-ray FEL pulses. J. Phys. B 39, L299-L304 (2006).

18. Sobierajski, R. et al. Experimental station to study the interaction of intense femtosecond vacuum ultraviolet pulses with matter at TTF1 free electron laser. Rev. Sci. Instrum. 76, 013909 (2005).

19. Chapman, H.N. et al. Femtosecond diffractive imaging with a soft-X-ray free-electron laser, Nature Physics 2, 839-843 (2006).

20. Altarelli, M. et al. (Eds.): XFEL: The European X-Ray Free-Electron Laser. Technical Design Report. Preprint DESY 2006-097, DESY, Hamburg, 2006 (see also http://xfel.desy.de).

21. Merkel, A. German Science Policy 2006. Science 313, 147 (2006).

22. Arthur, J. et al. Linac Coherent Light Source (LCLS). Conceptual Design Report. SLAC- R593, Stanford, 2002 (see also http://www-ssrl.slac.stanford.edu/lcls/cdr).

23. Tanaka, T. \& Shintake, T. (Eds.): SCSS X-FEL Conceptual Design Report. Riken Harima Institute, Hyogo, Japan, May 2005 (see also http://wwwxfel.spring8.or.jp).

24. Saldin, E.L., Schneidmiller, E.A. \& Yurkov, M.V. The physics of free electron lasers (Springer-Verlag, Berlin, 1999).

25. Bonifacio, R., Pellegrini, C. \& Narducci, L.M. Collective instabilities and high-gain regime in a free-electron laser. Opt. Commun. 50, 373 (1984).

26. Dohlus, M. et al. Start-to-end simulations of SASE FEL at the TESLA Test Facility, phase 1. Nucl. Instrum. and Methods A 530, 217-233 (2004).

27. Krinsky, S. \& Huang, Z. Frequency chirped self-amplified spontaneous-emission free-electron lasers. Phys. Rev. ST Accel. Beams 6, 050702 (2003).

28. Saldin, E.L., Schneidmiller, E.A. \& Yurkov, M.V. Selfamplified spontaneous emission FEL with energy-chirped electron beam and its application for generation of attosecond x-ray pulses. Phys. Rev. ST Accel. Beams 9, 050702 (2006).

29. Bytchkov, A. et al. Development of MCP-based photon diagnostics at the TESLA Test Facility at DESY. Nucl. Instrum. and Methods A 528, 254-257 (2004).

30. Richter, M. et al. Measurement of gigawatt radiation pulses from a vacuum and extreme ultraviolet freeelectron laser. Appl. Phys. Lett. 83, 2970-2972 (2003).

31. Saldin, E.L., Schneidmiller, E.A. \& Yurkov, M.V. Statistical properties of radiation from VUV and X-ray free electron laser. Opt. Commun. 148, 383-403 (1998).

32. Martins, M. et al. Monochromator beamline for FLASH. 
Rev. Sci. Instr. 77, 115108 (2006).

33. Colson, W. Free-electron lasers operating in higher harmonics. Phys. Rev. A 24, 639-641 (1981).

34. Freund, H., Biedron, S. \& Milton, S. Nonlinear harmonic generation in free-electron lasers. IEEE J. Quant. Electr. 36, 275-281 (2000).

35. Huang, Z, \& Kim, K. Three-dimensional analysis of harmonic generation in high-gain free-electron lasers. Phys. Rev. E, 62, 7295-7308 (2000).

36. Saldin, E.L., Schneidmiller, E.A. \& Yurkov, M.V. Properties of the third harmonic of the radiation from selfamplified spontaneous emission free electron laser. Phys. Rev. ST Accel. Beams 9, 030702 (2006).

37. Geloni, G., Saldin, E.L., Schneidmiller, E.A. \& Yurkov, M.V. Exact solution for second harmonic generation in
XFELs, Opt. Commun. 271, 207-218 (2007)

38. Saldin, E.L., Schneidmiller, E.A. \& Yurkov, M.V. FAST: a three-dimensional time-dependent FEL simulation code. Nucl. Instr. Meth. A 429, 233-237 (1999).

39. Tiedtke, K. et al. The SASE FEL at DESY: photon beam diagnostics for the user facility. AIP Conf. Proc. 705, 588592 (2004)

40. Nicolosi, P. et al. Grazing-incidence spectrometer for the monitoring of the VUV FEL beam at DESY. J. Electron Spec. Relat. Phenom. 144, 1055 (2005).

41. Kita, T., Harada, T, Nakano, N. \& Kuroda, H. Mechanically ruled aberration-corrected concave gratings for a flat-field grazing-incidence spectrograph. Appl. Optics 22, 512 (1983). 